\title{
Soil-plant transfer of polychlorinated biphenyls in paddy fields
}

\author{
Shaogang Chu*, Meilin Cai, Xiaobai Xu \\ Research Center for Eco-Environmental Sciences, Chinese Academy of Sciences, Beijing 100085, China
}

Received 15 November 1998; accepted 18 April 1999

\begin{abstract}
In a field experiment, the transfer of polychlorinated biphenyls (PCBs) from contaminated soil to rice was investigated. The results show that rice is not subject to significant bioconcentration of PCBs from the surrounding environmental system and the distribution of PCBs in the plant shows that the concentration series is leaves $>$ husk $>$ stalk $>$ rice. It is suggested that root uptake/translocation is not the main pathway of PCB contamination of rice, while atmospheric deposition might be the main pathway of most PCBs from soil to rice. Principal component analysis was performed with the standardized relative abundance of PCBs in the samples and main pollution source. The congener pattern of PCBs in the plant samples is not similar to those in soil and in fish. (C) 1999 Elsevier Science B.V. All rights reserved.
\end{abstract}

Keywords: PCBs; Rice; Soil; Transfer

\section{Introduction}

Polychlorinated biphenyls have been recognized for decades as one of the main chemical contaminants in water, sediments, soil and biota of the world. The environmental transfer and fate are of great importance, since use and disposal practices of existing PCBs must be controlled

\footnotetext{
* Corresponding author. Tel.: + 86-10-6292-5511, ext. 2157; fax: + 86-10-6255-5381.
}

based on sound prediction of their environmental impact.

It was generally agreed that human diet represents the main route of exposure to PCBs. Because of their ability to biomagnify through the food chain and our relative topmost position in the food chain, the occurrence of PCBs in various types of food has been widely studied. The route was believed to be responsible for human burden with meal, fish and milk, whereas the intake by consumption of vegetables was considered negligible. However, plants are the first link in the 
food chain and therefore it is necessary for full risk assessment to know by which pathway PCBs may be accumulated in plants.

PCBs are found in many environmental matrices, including marine plants. The accumulation and effects on plants were reviewed by Mahanty (1986) and Shimp et al. (1993). PCB accumulation in plants might be due to the following pathway: root uptake and transport to the shoot; contamination of shoots by soil particles and atmospheric deposition; and uptake of vapor phase by aerial plant parts. A more detailed knowledge about the contamination pathway is indispensable to determine critical soil levels and to recommend techniques for cultivation, harvest and pot-harvest processing of food crops. Experimental work with plant uptake of organic xenobiotics has been limited mostly to studies of herbicides and pesticides. Relatively little is known about plant uptake and metabolism of hydrocarbon and chlorinated compounds (Shimp et al., 1993). It was believed that PCBs were very immobile in soils and thus scarcely available for root adsorption or subsequent uptake. In field experiments, PCB concentration in shoots of various plant species was consistently very low. Current results of field studies show that PCB concentrations in plants were not correlated to the level of soil contamination, suggesting that atmospheric deposition was the main entry pathway of PCBs in plant shoots. In some experiments, no correlation between the PCDD/Fs concentration of the fruits and the corresponding soils was found. Higher levels of PCDD /Fs in peel than in pulp of fruits indicated that the atmospheric deposition was of far greater importance than the pathway of soil-plant (Muller et al., 1993). However, Hulster et al. have proved that for some plants, such as zucchini and pumpkin, root uptake of PCDD/PCDF and subsequent translocation to the shoots and into the fruits is the main contamination pathway (Hulster et al., 1994). These results suggested that different plants might perform differently with respect to the mechanisms of accumulating PCBs.

Rice is the main crop in the south of China. Not only is it the main food of humans, but it is also used widely to raise pigs, chickens and other livestock. An estimate of the amount of PCBs present in various ecological systems is important for the contaminated area and the remediation process. The main objective of the present study was an investigation, through field experiments, of the uptake of PCBs from contaminated soil by rice.

\section{Experimental}

\subsection{Sample collection}

Rice samples including rice, husk, stalk and leaves (including leaf sheath) were collected after harvest in October 1993 near a village where the field was polluted by PCBs. In order to investigate the real contamination by PCBs, no special attempt was made in harvest or cultivation process. The soil samples in the paddy field were collected from the 0 to $10-\mathrm{cm}$ layer, air-dried and sieved to $2 \mathrm{~mm}$. All samples were collected at the same time, packed in separate jars, and kept frozen until analysis.

\subsection{Sample extraction}

The analytical procedure for PCBs in soil is described in detail elsewhere (Chu et al., 1996) and briefly described here. An approximately 20 -g soil sample or rice sample was weighed, thoroughly mixed and ground with anhydrous sodium sulfate to flowing powder. The rice husk, leaves and stalk, approximately $10 \mathrm{~g}$, were cut with stainless-steel instruments to about $1 \mathrm{~mm}$ in length and ground with anhydrous sodium sulfate to flowing powder before extraction. Ultrasonic extraction was used to extract PCBs from the samples with $50 \mathrm{ml}$ hexane and acetone $(1: 1 \mathrm{v} / \mathrm{v})$ for $5 \mathrm{~min}$. After centrifugation the extract was separated from the residue. The extraction was repeated three times. The combined extract was concentrated to approximately $3 \mathrm{ml}$ by K.D. concentrator with a three-ball Snyder column on a steam bath.

\subsection{Clean up}

The sample solution was shaken with concen- 
trated $\mathrm{H}_{2} \mathrm{SO}_{4}$ in a test tube, after centrifugation the acid layer was discarded. This treatment was repeated several times until the hexane layer was clean. The hexane layer was washed with $2 \%$ $\mathrm{NaCl}$ aqueous solution, dried with anhydrous sodium sulfate and then concentrated to approxi-

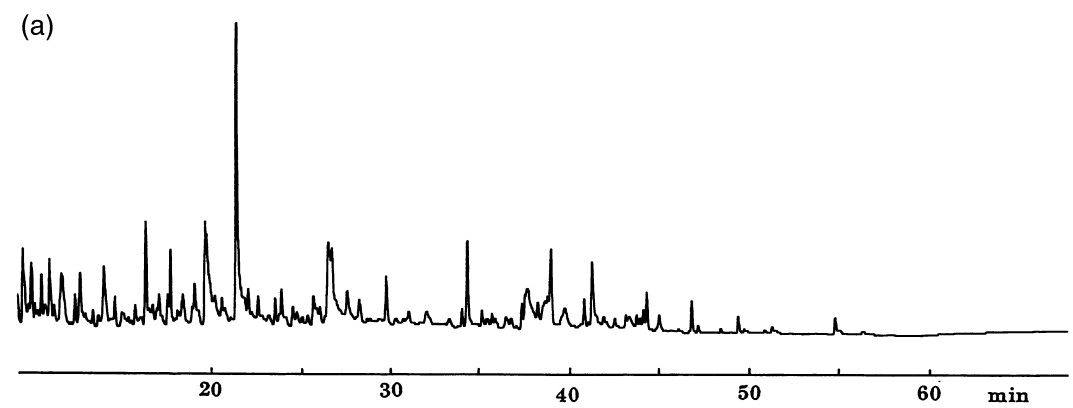

(b)

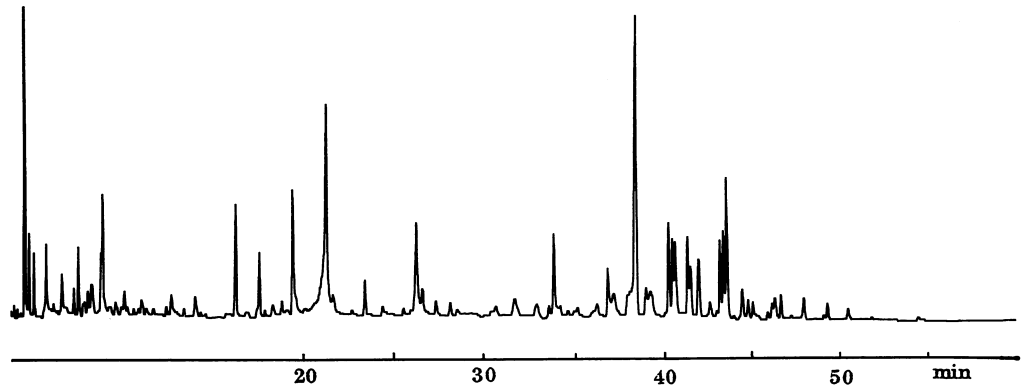

(c)

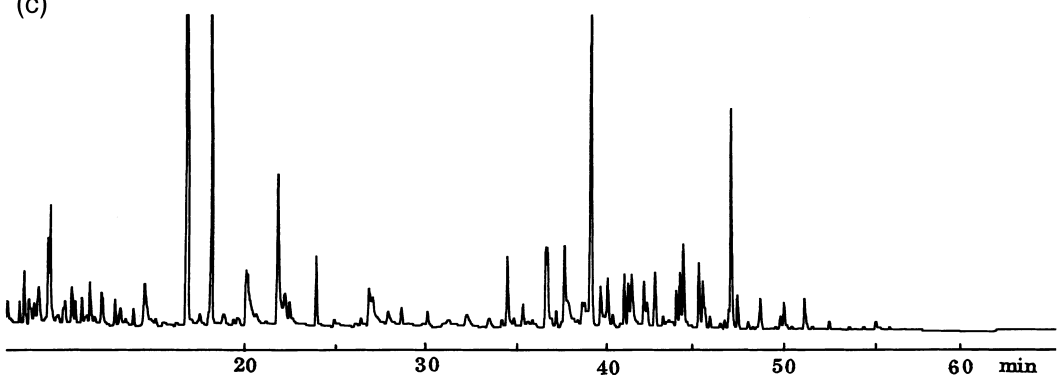

(d)

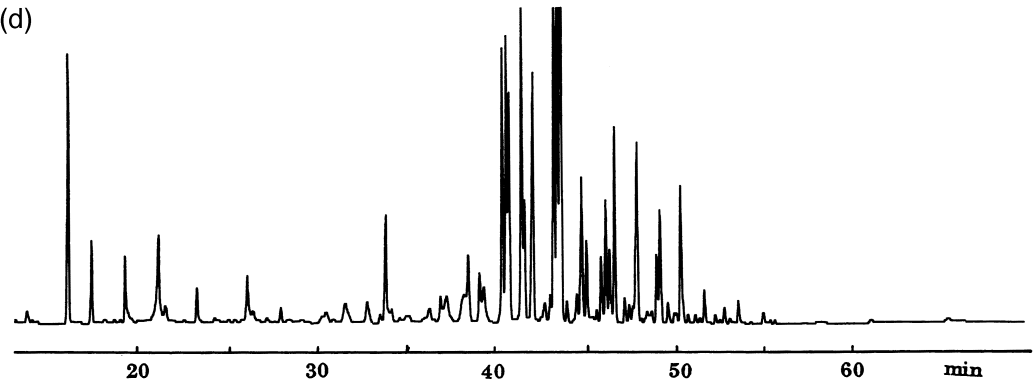

Fig. 1. 


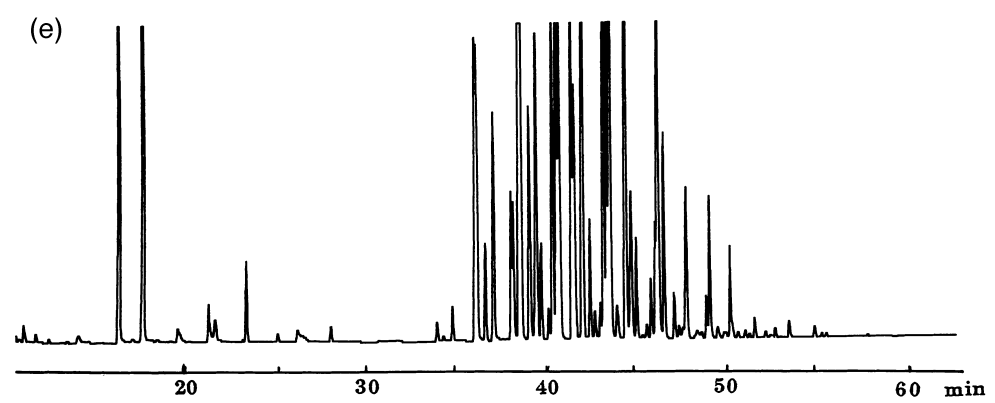

Fig. 1. (Contd.) GC/ECD chromatograms of PCB fraction in (a) rice, (b) stalk, (c) husk, (d) leaves and (e) soil.

mately $1 \mathrm{ml}$ for column chromatographic clean up.

Alumina $\left(\mathrm{Al}_{2} \mathrm{O}_{3}\right)-\mathrm{Ag}^{+} \quad(10 \%$ silver nitrate $)$ modified silica gel column chromatography separation was used in the second clean up step. The column, $300 \times 10 \mathrm{~mm}$ i.d. glass column with Teflon stopcock, was packed with $8 \mathrm{~g}$ basic alumina (deactivated by $2 \%$ water) and $5 \mathrm{~g}$ silica gel $(10 \%$ $\mathrm{AgNO}_{3}$ ), with $1 \mathrm{~g}$ anhydrous $\mathrm{Na}_{2} \mathrm{SO}_{4}$ placed at the top. After the sample was introduced, the column was eluted with hexane. The first $120-\mathrm{ml}$ hexane fraction was collected and concentrated to $0.2 \mathrm{ml}$ for gas chromatographic analysis.

\subsection{Gas chromatographic analysis}

Gas chromatography was performed with Varian 3740 equipped with a ${ }^{63} \mathrm{Ni}$ electron capture detector. The chromatograph was fitted with a SE-54 (40 $\mathrm{m} \times 0.25 \mathrm{~mm}$ i.d. J \& W Scientific Inc., USA). The carrier gas was nitrogen. The oven temperature was programmed as follows: initial temperature $50^{\circ} \mathrm{C}$ held for $2 \mathrm{~min}$, increased by $4^{\circ} \mathrm{C} \mathrm{min}{ }^{-1}$ to $280^{\circ} \mathrm{C}$, and held there for $10 \mathrm{~min}$. The temperatures of injector and detector were $300^{\circ} \mathrm{C}$ and $350^{\circ} \mathrm{C}$, respectively. A $1-\mu 1$ sample was injected to the chromatograph with splitless injection.

Data acquisition was accomplished through a Shimadzu Chromatopac C-R3A. Aroclor 1254 and 1242 (Supelco Inc.) mixture, the concentration of individual congeners were reported, were used as external standard calibration solutions for identification and quantification analysis (Capel et al., 1985). Calibration curves and limits of detection were determined by diluting a stock solution. The limit of detection was defined as the amount that gives a signal-to-noise ratio of 3 . For most congeners the limit of detection was $0.01 \mu \mathrm{g} / \mathrm{kg}$, but for PCB8 and PCB19, the limit of detection was $0.5 \mu \mathrm{g} / \mathrm{kg}$. The reproducibility of the analytical method averaged 9\% for total PCBs and the recovery was $83.8 \%$ and $78.2 \%$ for rice grain and soil sample, respectively, by spiking $2 \mu \mathrm{g}$ Aroclor 1254 in 20-g unpolluted samples. Blank analysis showed no interference peaks with the individual PCB congeners analysis.

Residual water was measured on subsamples $\left(24 \mathrm{~h}\right.$ at $\left.105^{\circ} \mathrm{C}\right)$ and results were calculated on dry weight.

\section{Results and discussion}

\subsection{Levels of contaminants}

Fig. 1 shows the GC/ECD chromatograms of PCB fraction in samples of rice, stalk, husk, leaves and soil. Table 1 shows the concentrations of individual PCB congeners in different samples.

Although there is a lot of data from investigations of pesticide contamination in rice, we have not found data from literature available with respect to PCBs in rice for direct comparison.

Arrifai investigated the contamination of PCBs in wheat, rye and barley harvested in 1972-1974 (Arrifai and Acker, 1975). The results showed that Clophen A60 was found in 76 samples at 0.01-0.13 ppm (air-dried grain), 57 samples had $<0.01 \mathrm{ppm}$, and only two samples were free of 
Table 1

Concentrations of PCBs in sample (ng/g dry wt.)

\begin{tabular}{|c|c|c|c|c|c|}
\hline IUPAC No. & Rice & Stalk & Husk & Leaves & Soil \\
\hline 8 & - & 0.51 & 0.90 & 1.0 & 0.85 \\
\hline 19 & - & - & - & - & 9.7 \\
\hline $18+17$ & 0.13 & 0.25 & 5.8 & 0.55 & 110.6 \\
\hline $16+32$ & 0.029 & 0.87 & 2.9 & 1.2 & 32.1 \\
\hline 26 & 0.37 & 0.30 & 0.70 & 1.8 & 15.6 \\
\hline 25 & - & 0.25 & 0.79 & - & 15.8 \\
\hline $31+28$ & 0.39 & 3.0 & 7.2 & 1.9 & 102.1 \\
\hline 33 & - & 0.60 & 1.6 & 2.1 & 36.4 \\
\hline 22 & - & 0.31 & 0.76 & 0.77 & 17.9 \\
\hline 45 & - & - & 0.42 & 0.079 & 12.8 \\
\hline 46 & 0.065 & - & 0.19 & - & 4.9 \\
\hline 52 & 0.26 & 1.7 & 2.2 & 11.6 & 73.0 \\
\hline 49 & 0.048 & 0.84 & 1.0 & 6.6 & 47.5 \\
\hline $47+48$ & 0.23 & 0.75 & 1.4 & 4.5 & 37.7 \\
\hline 44 & 0.043 & 1.1 & 1.6 & 9.6 & 58.4 \\
\hline $37+42$ & 0.081 & 0.68 & 0.79 & 3.4 & 36.2 \\
\hline $41+64$ & 0.041 & 0.81 & 1.6 & 6.8 & 65.0 \\
\hline 40 & - & - & 0.29 & - & 10.0 \\
\hline 74 & 0.11 & 0.82 & 1.0 & 8.3 & 46.5 \\
\hline 70 & 0.12 & 0.94 & 1.6 & 12.1 & 56.3 \\
\hline 66 & 0.19 & 1.6 & 2.4 & 19.7 & 72.5 \\
\hline $60+56$ & 0.10 & 0.36 & 1.2 & 0.64 & 37.9 \\
\hline 101 & 0.013 & 0.31 & 1.7 & 5.0 & 28.8 \\
\hline 99 & - & 0.33 & 0.52 & 3.3 & 16.3 \\
\hline 83 & - & - & - & 0.25 & 1.7 \\
\hline 97 & - & 0.13 & - & 2.1 & 9.4 \\
\hline 87 & - & 0.11 & 0.15 & 1.8 & - \\
\hline 85 & - & 0.25 & - & 1.6 & 63.8 \\
\hline $110+77$ & 0.08 & 0.31 & 1.2 & 5.3 & 26.9 \\
\hline 82 & - & 0.066 & 0.24 & 0.69 & 5.9 \\
\hline 151 & - & - & - & - & 1.9 \\
\hline $118+108$ & 0.036 & 0.32 & 0.98 & 5.9 & 17.9 \\
\hline 146 & - & 0.073 & 0.47 & 2.0 & 4.7 \\
\hline 153 & 0.04 & 0.084 & 0.34 & 1.3 & 5.6 \\
\hline 141 & - & - & 0.062 & 0.38 & 0.92 \\
\hline 137 & - & - & 0.030 & 0.14 & 0.18 \\
\hline 138 & - & 0.12 & 0.89 & 3.6 & 8.7 \\
\hline 178 & - & - & 0.20 & 0.34 & 1.0 \\
\hline 175 & - & - & - & 0.46 & 1.8 \\
\hline $187+159$ & - & - & 0.14 & 0.4 & \\
\hline 128 & - & - & 0.19 & 0.66 & 1.4 \\
\hline 185 & - & - & - & 0.14 & 0.48 \\
\hline 174 & - & - & - & 0.14 & 0.41 \\
\hline 177 & - & - & 0.10 & 0.37 & 0.93 \\
\hline 172 & - & - & - & - & 0.10 \\
\hline 180 & - & - & 0.069 & 0.35 & 1.1 \\
\hline 170 & - & - & 0.093 & 0.41 & 1.3 \\
\hline 196 & - & - & - & 0.097 & 0.36 \\
\hline 201 & - & - & - & 0.13 & 0.56 \\
\hline 195 & - & - & - & - & 0.46 \\
\hline 194 & - & - & - & 0.045 & 0.17 \\
\hline Total PCBs & 2.34 & 17.79 & 43.57 & 129.28 & 1099.62 \\
\hline
\end{tabular}

PCBs. Three samples also contained Clophen A30 with a mean of $0.21 \mathrm{ppm}$. There was no detectable trend between concentration and year of harvest. Schecter et al. determined PCDD/Fs, PCBs and DDE in pooled food samples collected in 1995 at supermarkets across the United States. The results showed that the concentration of coplanar, mono- and di-ortho-PCBs in simulated vegan diet sample is $159 \mathrm{ppt}$ (Schecter et al., 1997). Compared with the concentration of the PCBs in fresh fish and eggs (7481 and 1536 ppt) and our data of PCBs in fish and egg samples collected nearby the paddy field (846 ppb and 21 $\mathrm{ppb}$ ), the concentration of PCBs in vegetables is very low. It seems that the rice does not specifically bioconcentrate PCBs from the environmental system like several other plants, e.g. PCDD/ PCDF concentrations were approximately two orders of magnitude higher in the cucumber family than in other fruits and vegetables (Hulster et al., 1994).

\subsection{Atmospheric deposition as a possible pathway of contamination}

The PCBs contamination in plant biota such as tree leaves was widely measured because it may be useful for indirect quantitative long-term air monitoring if the age of leaves, sampling location and other factors to be controlled (Morosini et al., 1993). There are a lot of data about the distribution of PCBs in survey of pine needles (Tremolada et al., 1996). Many models have been set up for the accumulation of pesticides and other contaminates in the air-soil-plant leaf. Multicompartment models for uptake of organic pollutant, including PCBs, in the air-soil-plant have been developed (Trapp et al., 1994). Octanol-air partition coefficients $\left(K_{\mathrm{OW}}\right)$ are proposed as valuable descriptors of air-vegetation and air-soil equilibrium (Harner and Mackay, 1995). An archive of annual harvests of pasture grass was compared with archived air filters, and a general correlation of PCB levels was found by Jones et al. (1995). They used the data to document declining air levels of PCBs in rural UK indicating there were two main sources of PCBs to plants: atmosphere and soil. PCBs are highly 
lipophilic, with the consequence that more than $90 \%$ of PCBs mass was found in soil, but it was generally considered that in soil PCBs were very immobile and thus scarcely available for root adsorption or results in measurable atmospheric emission. Eisenreich estimated that the atmospheric pathway contributes $60-90 \%$ of PCB input into the Great Lakes, and Lake Superior receives more than $78 \%$ of the PCB burden from the atmosphere (Eisenreich, 1987). According to this fact, the possibility of volatilization of PCBs from soil and subsequent adsorption to plant was suggested.

The previous works succeeded in demonstrating that root uptake/translocation is not relevant for the accumulation of PCBs in beans, broad beans, tomatoes and cucumbers (Bacci and Gaggi, 1985). That PCBs move slowly within tomato plants has been proved directly. Ninety-five percent of injected tetra- and hexa-CB was recovered within $5 \mathrm{~cm}$ of the injection point after 55 days and no metabolism was detected (Ye et al., 1992). A translocation study with corn and goldenrod plants shows that less than $1 \%$ of the ${ }^{14} \mathrm{C}$-labelled Aroclor 1242 entering the roots is translocated to aboveground portions of these plants over an entire growing season (Buckley, 1982).

This field study also supports that this suggestion is applicable to rice plants. In the samples, the concentration of PCBs in leaves is higher than in husks and is much higher than in stalks. If rice plants take up PCBs mainly from the roots and uptake/translocate them to other tissue, the concentration of PCBs in stalk should not be lower than in leaves. The atmospheric deposition hypothesis could explain the distribution of PCBs in rice plant tissues. In this situation, the accumulation in plants can be expressed as the time integral of the product of gaseous concentration, and airside deposition velocity and the surface area (McLachlan, 1996). Leaves are exposed to the atmosphere and can concentrate the contamination from dry gaseous deposition; dry particle bound deposition and wet deposition. In comparison with leaves, the husk has the relatively smaller rate of surface area/volume than leaves, so the concentration of PCBs in the husk is lower than in leaves. Although rice stalks have the longest live time in the rice-growing season, most of the rice stalk is wrapped by leaf sheath, with only about one-tenth of the stalk exposed to the atmosphere. The relatively smaller rate of surface area/volume, as our results indicate, explains how the concentration of PCBs in stalk is much lower than in rice leaves.

\subsection{Results of principal component analysis}

Principal component analysis (PCA) was widely used to differentiate $\mathrm{PCB}$ congener patters in environmental samples. If the root uptake/translocation is not relevant for the accumulation of $\mathrm{PCBs}$ in rice, the distribution of individual $\mathrm{PCB}$ congener in rice tissues is dependent on the vapor patterns, but not lipophilicity. Physical properties such as water solubility, vapor pressure, and Henry's law constant suggest that PCBs have a different distribution in different environmental samples. To study the covariation of several parameters and the grouping of the samples, principal component analyses were performed with the standardized relative abundance of the congeners in the samples and main pollution source. The principal component analysis was carried out using SAS 6.12 software. The data were normalized, divided by the sum of PCB concentration in each sample. The concentration of individual congeners below the detection limit was set to zero. In this case, the first four principal components 1(PC1), 2(PC2), 3(PC3) and 4(PC4) are 52.0, 15.3, 12.2 and $8.0 \%$, respectively, of the total variability. Fig. 2 shows the first two components of the principal component analysis, which contained $67.3 \%$ of the total variability. PC1 is chiefly determined by the contribution of the total chlorine in the congeners. Most di- and tri-chlorobiphenyls present a negative value and hexa- and heptachlorobiphenyl present a positive value. It seems that the $\mathrm{PC} 1$ reflects the distribution of the total chlorinated of the PCBs in the samples. PC2 is chiefly determined by the contribution to congeners 52, 74, 66, 47 and 49 with negative values of $-0.328,-0.327,-0.283,-0.249$ and -0.232 and congeners $40,47,45,18$ and 33 , with positive values of $0.261,0.249,0.212,0.188$ and 0.186 , respectively. Most of them are tetra-chlorobi- 
phenyl with two other chlorine atoms in two benzene rings. It seems that reflected the different pathways of PCB entry. Leaves have a higher value, while rice has the lowest value of principal component 2; the other samples, husk and stalk, occupy intermediate positions. China produced PCB products with the tradenames of $\mathrm{PCB}_{3}$ and $\mathrm{PCB}_{5}$. According to the previous work and this $\mathrm{PCA}, \mathrm{PCB}_{3}$ is very similar to Aroclor 1242 , while $\mathrm{PCB}_{5}$ is somewhat close to Aroclor 1254. In the previous investigation the main contamination in the area was $\mathrm{PCB}_{3}$. Nearby the paddy field, some farmers destroyed a few capacitors and transformers, which used $\mathrm{PCB}_{3}$ as dielectric fluids. If the rice plant surface contamination by soil particles was the main source of pollution, the congener distribution in the plant samples should be similar with the pattern in soil, or if so it means that the rice sample points in the first two components of the principal component analysis should be much more close to the point of $\mathrm{PCB}_{3}$. In fact this situation is not found in the PCA. It means that the PCBs contamination in rice plant does not come from the soil directly in the cultivation or harvesting process. Therefore atmospheric deposition is suggested as the main entry pathway of PCBs in rice. To compare the plant sample with others, the PCA was also used in the fish sample. In the PCA, fish present markedly different. This difference is probably related to the routes of the different bioaccumulation and the metabolism of fish.

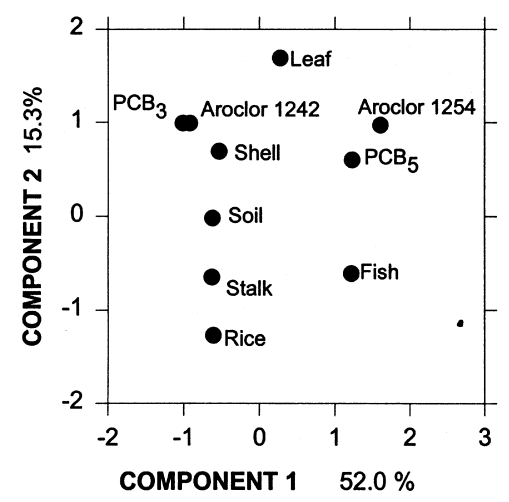

Fig. 2. Representation of the first two components of a principal component analysis in the paddy field samples.

\subsection{Other environmental impacts}

Though the concentration of PCBs in rice is much lower than in fish and soil, it should be mentioned that in the south of China, rice straw is usually used for cooking and sometimes farmers burn rice straw in fields to remove it for the next planting. Risk assessments on polychlorinated dibenzo- $p$-dioxins and polychlorinated dibenzofurans in combustion sources and environments are being conducted in various countries. Rice straw burning is known to cause localized deterioration of air quality, which is most often due to particulate matter released from rice straw burning. Muto et al. have studied PCDD/Fs in rice straw smoke generated by laboratory burning experiments (Muto et al., 1993). It was proved that PCDD/Fs were produced by the straw burning and transferred to the smoke. Straw burning is mostly performed under incomplete combustion, and the atmosphere surrounding the place suffers serious pollution by the smog. It is suggested that the additional health hazards to PCB exposure will be present which is associated with the burning season of rice straw. Further study on PCDD/F formation by the burning is necessary in order to assess the unrecognized health effects by rice straw smoke.

\section{Acknowledgements}

This work was funded by the Research Foundation of Chinese Academy of Sciences (Bureau of Resources and Environment and 98 Director's Fund of Bureau of Basic Studies).

\section{References}

Arrifai K, Acker L. The contamination of grain with polychlorinated biphenyls and hexachlorobenzene. Ber Getreidechem-Tag Detmold, 1975:103-110.

Bacci E, Gaggi C. Polychlorinated biphenyls in plant foliage: translocation or volatilization from contaminated soils? Bull Environ Contam Toxicol 1985;35:673-681.

Buckley EH. Accumulation of airborne polychlorinated biphenyls in foliage. Science 1982;216:520-522.

Capel PD, Rapaport RA, Eisenreich SJ, Looney BB. PCBQ: computerized quantification of total $\mathrm{PCB}$ and congeners in environmental samples. Chemosphere 1985;14:439-450. 
Chu S, Yang C, Xu X. Determination of polychlorinated biphenyl congeners in environmental samples. J Environ Sci 1996;8:57-65.

Eisenreich SJ. The chemical limnology of nonpolar organic contaminants: polychlorinated biphenyls in Lake Superior. Adv Chem Ser 1987;216:393-469.

Harner T, Mackay D. Measurement of octanol-air partition coefficients for chlorobenzenes, PCBs, and DDT. Environ Sci Technol 1995;29:1599-1606.

Hulster A, Muller JF, Marschner H. Soil-plant transfer of polychlorinated dibenzo- $p$-dioxins and dibenzofurans to vegetables of the cucumber family (Cucurbitaceae). Environ Sci Technol 1994;28:1110-1115.

Jones KC, Duarte-Davidson R, Cawse PA. Changes in the PCB concentration of United Kingdom air between 1972 and 1992. Environ Sci Technol 1995;24:272-275.

Mahanty HK. Polychlorinated biphenyls; accumulation and effects upon plants. In: Waid JS, editor. PCBs and the environment, vol 2. Boca Raton, Fl: CRC Press, 1986:1-8.

McLachlan MS. Bioaccumulation of hydrophobic chemicals in agricultural food chains. Environ Sci Technol 1996;30: 252-259.

Morosini M, Schreitmuller J, Reuter U, Ballschmiter K. Correlation between C-6/C-14 chlorinated hydrocarbons levels in the vegetation and in the boundary layer of troposphere. Environ Sci Technol 1993;27:1517-1523.
Muller JF, Hulster A, Papke O, Ball M, Marschner H. Transfer pathways of PCDD/PCDF to fruits. Chemosphere 1993; 27:195-201.

Muto H, Saitoh K, Takizawa Y. Polychlorinated dibenzo-p-dioxins and dibenzofurans in rice straw smoke generated by laboratory burning experiments. Bull Environ Contam Toxicol 1993;50:340-347.

Schecter A, Cramer P, Bogges K, Stanly J, Olson JR. Levels of dioxins, dibenzofurans, PCB and DDE congeners in pooled food samples collected in 1995 at supermarkets across the United States. Chemosphere 1997;34:1437-1447.

Shimp JF, Tracy LC, Davis LC, Lee WE, Huang W, Erickson LE, Schoor JL. Beneficial effects of plants in the remediation of soil and groundwater contaminated with organic materials. Crit Rev Environ Sci Technol 1993;23:41-77.

Trapp S, Mcfarlane C, Matthies M. Model for uptake of xenobiotics into plants: validation with bromacil experiments. Environ Toxicol Chem 1994;13:413-422.

Tremolada P, Burnett V, Calamari D, Jones K. A study of the spatial distribution of PCBs in the UK atmosphere using pine needles. Chemosphere 1996;32:2189-2203.

Ye Q, Puri RK, Kapila S, Yanders AF. Studies on the transport and transformation of PCBs in plants. Chemospere 1992;25: 1475-1479. 